\title{
Transcriptome analysis of primary chicken cells infected with infectious bronchitis virus strain K047-12 isolated in Korea
}

\author{
Rangyeon Lee ${ }^{1} \cdot$ Ji Seung Jung ${ }^{1} \cdot$ Ji-in Yeo ${ }^{1} \cdot$ Hyuk Moo Kwon ${ }^{1} \cdot$ Jeongho Park ${ }^{1}[$
}

Received: 2 February 2021 / Accepted: 15 April 2021 / Published online: 5 June 2021

(c) The Author(s), under exclusive licence to Springer-Verlag GmbH Austria, part of Springer Nature 2021

\begin{abstract}
Infectious bronchitis virus (IBV), an avian coronavirus, is highly contagious. Chickens with IBV infection develop acute pathogenesis in multiple organs, including the respiratory and urogenital tracts. Frequent recombination in the spike (S) glycoprotein gene has made vaccine strategies ineffective. To understand IBV pathogenesis, we analyzed the genetic distance between Korean IBV isolates and other coronaviruses, including SARS-CoV-2. To obtain comprehensive information about early immune responses such as innate cytokine production and associated immune regulation during IBV infection, we infected primary chicken embryonic kidney cells and performed transcriptome analysis. We observed that the functional pathways of innate immunity are regulated and confirmed expression of genes that coordinate early immune responses. Understanding the immune profile of the host cell may assist in vaccine development.
\end{abstract}

\section{Introduction}

Avian infectious bronchitis virus (IBV) is an important coronavirus that causes infectious bronchitis (IB) in the upper respiratory tract and spreads to remote organs $[1,2]$. IBV is classified as a gammacoronavirus, while SARS-CoV-2 is classified as a betacoronavirus. Swine coronaviruses such as porcine epidemic diarrhea virus (PEDV) and porcine deltacoronavirus (PdCV) are classified as alpha- and deltacoronaviruses, respectively [3].

The spike (S) glycoprotein mediates entry of the coronavirus into the host cell. It is a major inducer of host immune responses and is a determinant of host range and cell tropism [3-5]. The $\mathrm{S}$ glycoprotein is cleaved into $\mathrm{S} 1$ and $\mathrm{S} 2$ subunits. The S1 protein facilitates viral attachment to the host cell receptor, and the $\mathrm{S} 2$ protein mediates the fusion between the viral and host cell membranes $[4,6]$. Genetic modifications and recombination within the $\mathrm{S} 1$ coding region result in the generation of new viral subtypes. Consequently, vaccination

Handling Editor: Sheela Ramamoorthy.

Jeongho Park

jhp@ kangwon.ac.kr

1 College of Veterinary Medicine and Institute of Veterinary Science, Kangwon National University, Chuncheon, Gangwon-do, Republic of Korea is ineffective because genetic variations result in poor crossprotection $[1,2,7]$.

When IBV exhibits renal tropism, it replicates in the tubules and induces interstitial nephritis and tubular pathology [8-10]. We previously reported the genetic features of a Korean IBV isolate (K047-12) that causes renal atrophy [11], and the current study was designed to analyze the host immune response to this strain. Using phylogenetic analysis of S1 protein sequences, we also examined the genetic relationship of IBV to other coronaviruses. Using chicken embryonic kidney (CEK) cells, we investigated changes in various immune pathways during acute infection with K04712 by transcriptome analysis. Our results provide information about the role of host immune responses in renal pathogenesis at the cellular level when IBV affects kidney tissue. Moreover, understanding the interaction between avian coronaviruses and their hosts can contribute to preparation for the potential emergence of cross-species viral variants.

\section{Materials and methods}

The IBV K047-12 strain was isolated and propagated as reported previously [11]. Briefly, the virus was isolated in 2012 from the cecal tonsil of a 6-week-old layer in the city of Pocheon, Gyeonggi Province, Korea. The virus was transmitted via the in ovo route to 9- to 11-day-old specificpathogen-free (SPF) chicken embryos. Before infecting CEK 
cells, the virus was passaged three times in SPF embryonated eggs (Sungmin Farm, Korea).

Six representative coronaviruses and three IBV strains were chosen for this study. The nucleotide sequences of the $S$ glycoproteins were downloaded from the NCBI GenBank database as follows: HCoV-NL63 (NC_005831.2), TGEV (NC_038861.1), PEDV (NC_003436.1), SARS-CoV-2 (NC_045512.2), Bat-SL-CoVZXC21 (MG772934.1), IBV Beau-R (AJ311317.1), IBV M41-CK (MK728875.1), IBV K047-12 (MK618759.1), and PDCV (MN058072.1). S gene sequences were aligned by the Clustal $\mathrm{W}$ method using MEGA X software (version 10.2.2). A phylogenetic tree was constructed by the neighbor-joining method with 1,000 bootstrap replicates, using MEGA X software [12].

Next, CEK cells were prepared for transcriptome analysis by adapting a previously reported method [13]. Kidney tissues were collected from 18-day-old SPF eggs. Primary CEK cells were obtained by homogenization and seeded into 12 -well plates $\left(1 \times 10^{6}\right.$ cells/well $)$ in $1 \mathrm{ml}$ of DMEM medium with $10 \%$ FBS. Cells were divided into a K04712-infected group and an uninfected control group and cultured for 4-5 days until a monolayer was formed ( $90 \%$ confluence). Cells were infected by replacing half of the medium with prewarmed medium containing an IBV virus suspension, and an equal amount of fresh medium without virus was used for the control cells. Cells were harvested at 48 hours postinfection and stored in TRIzol Reagent (Ambion, Austin, TX, USA) for extraction of total RNA. We repeated the infection experiments six to seven times, and the extracted RNA from a representative preparation was sent to Macrogen (Seoul, Korea) for RNA sequencing. The RNA was purified, fragmented, reverse transcribed, and amplified by PCR for sequencing. The expression levels of transcripts were calculated as the fragments per kb of transcript per million mapped reads (FPKM). The filtered data were normalized by trimmed mean of m-value (TMM) normalization using the calcNormFactors method in the edgeR R library [14-17]. The criteria for significant differential expression of genes between the two groups were as follows: fold change $\geq 1.5$, raw $p$-value $<0.05$. The statistical significance of differences in expression levels was determined using exactTest within edgeR. Gene ontology (GO) and functional annotation analysis for the DE genes was performed using gProfiler [18]. The significant data were analyzed using KEGG pathway enrichment. The transcriptome data for IBV strains (M41-CK, Beau-R, K047-12) were analyzed by hierarchical clustering (Euclidean distance and average linkage). The analysis was performed using Cluster 3.0 software and Java TreeView 1.2.0. The differential expression data for the M41-CK and Beau-R strains were obtained from a recent study [1].

For PCR, RNA was first reverse transcribed into cDNA using AccuPower RT mix (Bioneer, Daejeon, Korea) according to the manufacturer's protocol. Quantitative PCR was then carried out in a $20-\mu \mathrm{L}$ reaction mixture using Maxima SYBR Green/ROX qPCR Master Mix (Thermo Fisher, Waltham, MA, USA). All reactions were carried out in duplicate. The reaction conditions were $50^{\circ} \mathrm{C}$ for $2 \mathrm{~min}$, $95^{\circ} \mathrm{C}$ for $10 \mathrm{~min}$, and 40 amplification cycles consisting of $95^{\circ} \mathrm{C}$ for $15 \mathrm{~s}$ and $55^{\circ} \mathrm{C}$ ( $\beta$-actin, IL-1 $\beta$, IL-10, IL-12a, TLR4, TLR7, CCR2, CCR7) or $50^{\circ} \mathrm{C}$ (IL-6R, IL-12RH, IL1RAP) for $1 \mathrm{~min}$. The melt curve stage was performed at $95^{\circ} \mathrm{C}$ for $15 \mathrm{~s}, 60^{\circ} \mathrm{C}$ for $1 \mathrm{~min}$, and $95^{\circ} \mathrm{C}$ for $15 \mathrm{~s}$. Threshold cycle $(\mathrm{Ct})$ values were determined, and relative gene expression levels were calculated by the $2^{-\Delta \Delta \mathrm{CT}}$ method. mRNA levels were normalized against $\beta$-actin. Ten genes associated with immune responses were selected for validation at the mRNA level. Primers were designed based on published chicken genome sequences in the NCBI database. The primer sequences were obtained from published papers or designed using a primer design tool (Primer 3 and BLAST, NCBI) and are listed in Table 1.
Table 1 Primers used for qRTPCR

\begin{tabular}{llll}
\hline Target & Forward primer (5'-3') & Reverse primer (3'-5') & Reference \\
\hline$\beta$-actin & TTGTCCACCGCAAATGCTTC & AAGCCATGCCAATCTCGTCT & This study \\
$I l-1 \beta$ & ACAGAGATGGCGTTCGTTCCCGA & TCAGCTCGACGCTGTCGATGT & {$[49]$} \\
$I l-10$ & CTGTCACCGCTTCTTCACCT & ATCAGCAGGTACTCCTCGAT & {$[50]$} \\
$I l-12 \alpha$ & CAGAGCTGGGGAACCTCAAG & CATCTCTGCAGTGAGGGCAC & This study \\
Tlr 4 & TCTTTCAAGGTGCCACA & GGATATGCTTGTTTCCAC & This study \\
$T l r 7$ & TCTGGACTTCTCTAACAACA & AATCTCATTCTCATTCATCATC & {$[51]$} \\
$C c r 2$ & ATGCCAACAACAACGTTTGA & TGTTGCCTATGAAGCCAAA & {$[52]$} \\
$C c r 7$ & CGGCTGAAGACCATGACAGA & CAGCCTGAACGATGGCAAAG & This study \\
$I l 6 r$ & CGCCTGCTGGTGGAAGA & TTCACCCGGCAGACGAATTT & This study \\
Il $r g$ & CTACGTGCGCAGCAAGATCA & GACACTGTGAGGACACTGCGG & This study \\
Ill rap & TCGTGGATGCCAAAGTCACA & AGTGCTTGAGGCCGAATCTT & This study \\
\hline & & &
\end{tabular}




\section{Results}

Sequences from the GenBank database were used to make a multiple alignment, and a phylogenetic tree was constructed to determine the genetic relationships between IBVs and other coronaviruses. We observed that the S1 protein sequences of IBV strains, including the Korean IBV isolate (K047-12), showed more similarity to those of SARS-CoV-2 and bat coronaviruses than to those of alpha- and deltacoronaviruses (Fig. 1A). Because the K047-12 strain causes renal damage, we infected primary CEK cells with the virus and performed RNA sequencing to investigate changes in expression of cellular genes. Out of 17,026 total genes examined, we identified 1,084 genes whose expression showed a $>1.5$-fold change. Of those genes, 787 were upregulated and 297 were downregulated (Fig. 1B).

The differentially expressed genes were classified using GO enrichment analysis, which suggested significant changes in biological processes and molecular functions. In the analysis of biological processes, K047-12 infection significantly altered the expression of about 150 genes involved in leukocyte activation, inflammatory responses, and cytokine-mediated signaling pathways. Analysis of molecular functions revealed that pathways for cytokine and immune receptor activity were highly regulated (Fig. 2A). In addition, we examined functional classification by KEGG pathway analysis. In accordance with the GO enrichment analysis, the top-ranked functions were closely correlated
Fig. 1 Phylogenetic analysis of IBV 047-12 and identification of differentially expressed genes during infection. (A) Neighborjoining phylogenetic tree based on $\mathrm{S} 1$ sequences. The percentage of replicate trees in which the associated taxa clustered together in the bootstrap test is shown next to each branch. The tree is drawn to scale, with branch lengths in the same units as those of the genetic distances used to infer the phylogenetic tree. The IBV strains are highlighted. (B) Volcano plot showing $p$-values for comparisons of $\log 2$-fold change in expression level between control cells and infected cells. The blue color represents downregulated genes, and the yellow bar represents upregulated genes (left). The number of differentially expressed genes is shown in the histogram (right). The criteria for significant differential expression were as follows: fold change $\geq 1.5$, raw $p$-value $<0.05$.
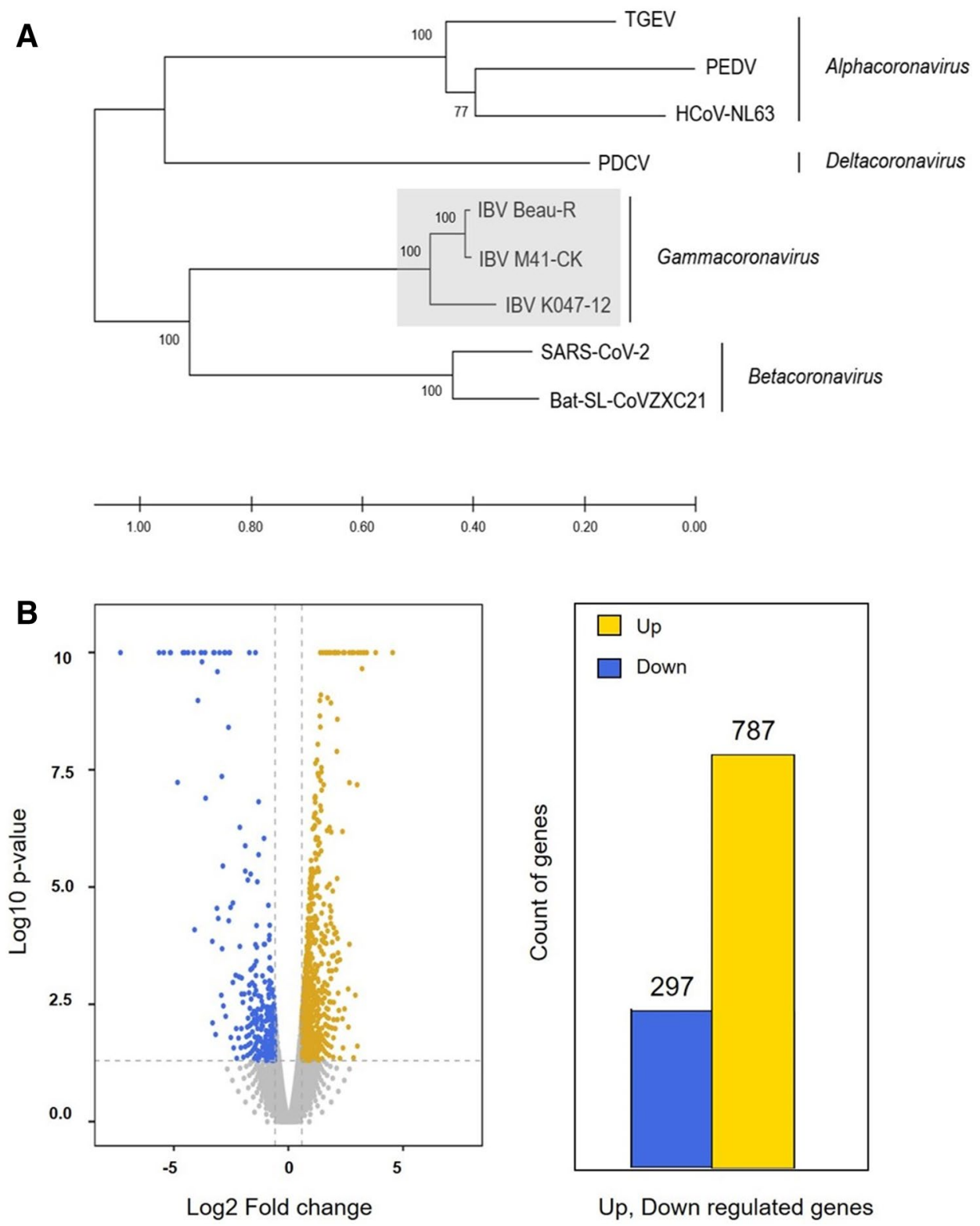

Up, Down regulated genes 

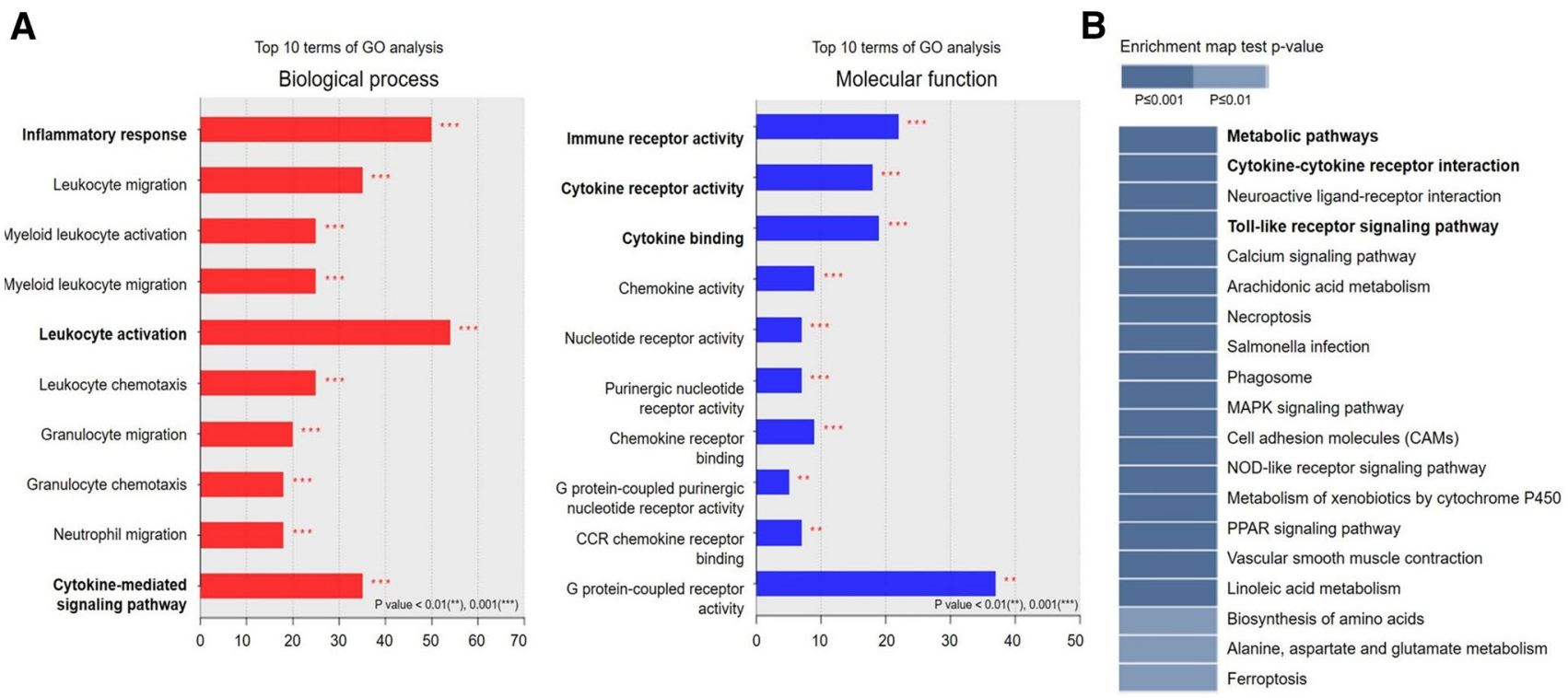

Fig. 2 Functional classification of differentially expressed genes. shown within biological processes and molecular function. (B) Heat map showing the results of gene-set enrichment analysis using a (A) Classification by GO enrichment analysis. The top 10 terms are

modified Fisher's exact test on each pathway map. The legend shows $p$-values, where a $p$-value $<0.05$ indicates a significantly enriched pathway term.

validates the transcriptome analysis and shows that renal cells are a source of critical immune regulators during the early stage of IBV infection.

ways, cytokine-cytokine receptor interactions, and the Tolllike receptor (TLR) signaling pathway (Fig. 2B). The functional classification demonstrates that K047-12 infection of CEK cells activates the immune system.

To identify strain-specific effects of the K047-12 strain on the host immune system, we compared our gene expression data with those obtained previously with other IBV strains. We retrieved the raw data for gene expression in primary renal cells that were infected with M41-CK or Beau-R in a previous study [1]. We then clustered genes by their functions in the immune response, cytokine responses, and cell activation. As shown in Fig. 1A, the S1 nucleotide sequence of M41-CK is more similar to that of Beau-R than to that of the K047-12 strain. However, the pattern of genes activated by K047-12 infection was more similar to that of M41-CK than to that of Beau-R (Fig. 3A). This suggests that the immune response is affected by factors other than the $\mathrm{S} 1$ sequence. Based on their functional classification, we identified highly regulated genes associated with innate immunity (Table 2). To verify the transcriptome analysis, we designed primers for 10 genes and confirmed their expression at the mRNA level. Following K047-12 infection, the levels of IL-1 $\beta$, IL-10, IL-1RAP, and IL-2RG for inflammatory signaling, TLR4 and TLR7 for RNA-virus-specific pattern-recognition receptors (PRRs), and CCR2 and CCR7 for lymphocyte migration increased significantly (Fig. 3B). In contrast, IL-12a and IL-6R expression did not change significantly but did show an increasing trend. This result
Complete prevention of IB by vaccination has not been very successful because of the rise of variant virus strains and unsuccessful vaccination strategies using inefficient routes, low vaccine titers, partial inactivation, and interference between vaccine strains $[19,20]$. The respiratory track is the primary organ affected by IBV infection, but genetic recombination in the $\mathrm{S} 1$ coding region expands the range of remote tissues that can be infected [10,21,22]. Earlier studies on IBV focused more on viral activity and genetic features than on host-pathogen interactions [23-25]. Recently, we isolated an IBV strain that showed kidney tropism and reported its genetic characteristics by comparing IBV isolates from different regions [11]. In the current study, we examined the genetic relationship of the K047-12 strain to coronaviruses from different host species. Interestingly, IBV showed a relatively close relationship to betacoronaviruses such as SARSCoV-2 and bat coronaviruses. Considering the repeated emergence of new variants amid the coronavirus pandemic (COVID-19), we speculate that a zoonotic coronavirus could arise through mutation of IBV. We therefore investigated how host cells respond to IBV infection and regulate their innate immune response. Infection by kidney-adapted IBV is

\section{Discussion}


Fig. 3 Classification of differentially expressed genes by immunologic function. (A) Hierarchical clustering of differential expression profiles of genes expressed in cells infected with strains K047-12, M41-CK, and Beau-R. Gene profiles were classified based on GO enrichment. The diagram indicates the correlation of the gene expression levels from all samples compared against each other. Colors represent the relative expression levels of genes, where yellow and blue indicate higher and lower expression, respectively (white color for no change). (B) Expression of genes involved in innate immunity, demonstrated by qRTPCR. Cytokine and receptorrelated molecules (upper) and TLRs and chemokine receptors (lower) expressed in primary chicken kidney cells are shown. Combined data from 6-7 experiments are shown, and error bars represent SEM. *, $p \leq 0.05$ from uninfected groups by nonparametric and paired $t$-test

\section{A}

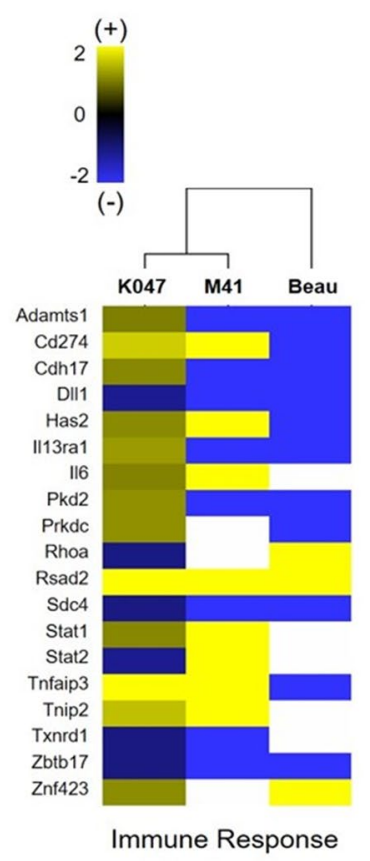

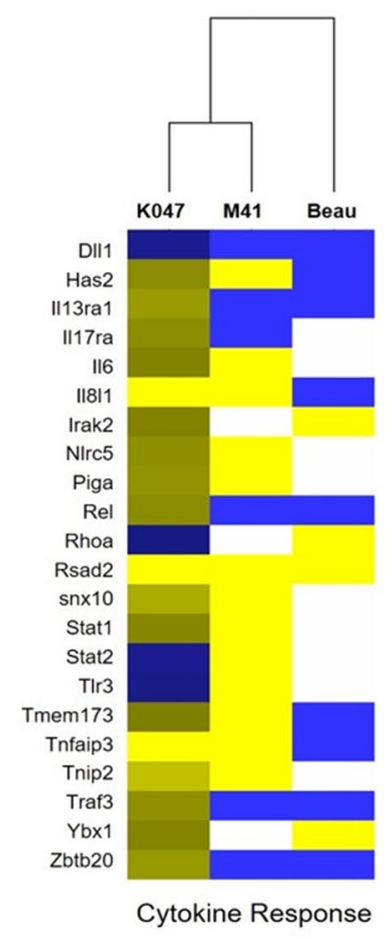

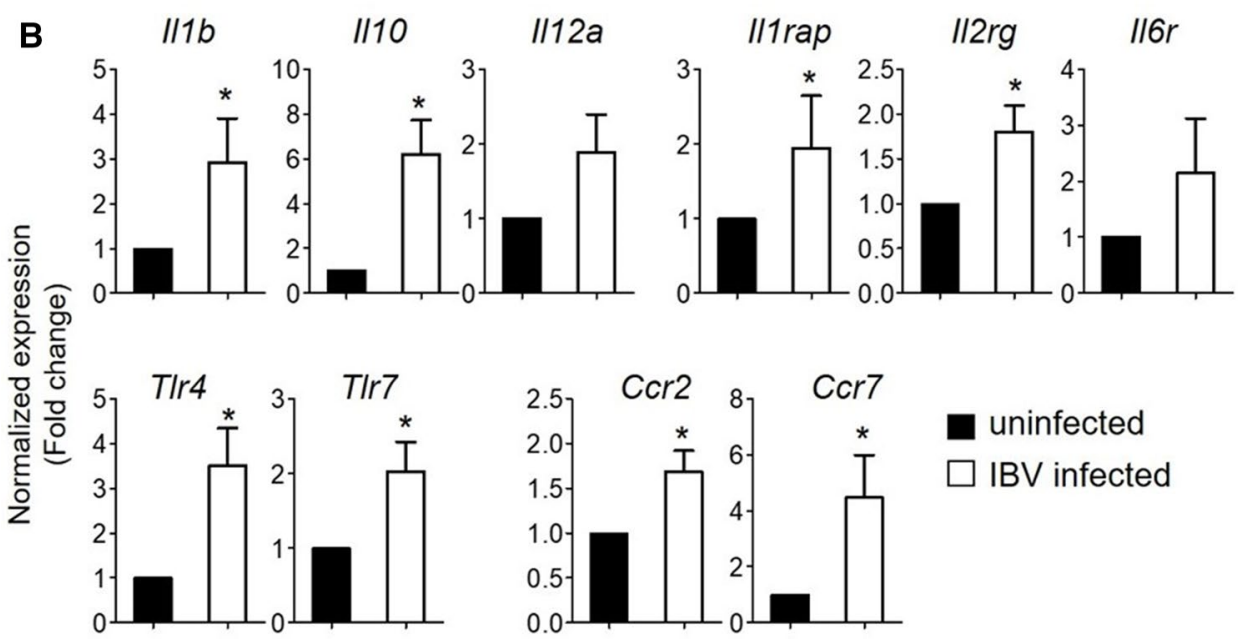

harmful to chickens and results in systemic pathology. Thus, the transcriptome analysis of kidney tissue infected by an IBV strain with renal tropism is helpful for understanding the pathogenesis of infectious bronchitis [8, 26, 27]. As the first line of defense, innate immunity is activated within two days after antigenic stimulation [28]. At this initial stage of IBV infection, cytokines and chemokines are produced and co-stimulatory pathways are activated $[29,30]$. To gain more precise information about the regulation of genes involved in innate immunity during kidney infection, we infected CEK cells with IBV isolate K047-12 for 48 hours and measured changes in the expression of genes associated with immune functions. The expression of 1,084 genes was found to change significantly; 787 genes were upregulated and 297 were downregulated. Assuming that the upregulated genes are linked to initial immune responses, we determined their functional significance using the GO enrichment and the KEGG pathway programs.

Functions for innate immunity regulation, such as leukocyte activation, inflammatory response, and cytokinemediated signaling pathways, were activated by K047-12 infection. A recent study using the M41-CK strain showed a comparable gene regulation pattern [1]. For example, the RSAD2, SNX10, STAT1, TNIP2, IL-6, IL-8L1, and DLL1 genes, which are associated with cytokine pathways, were upregulated by infection with kidney-adapted IBV. Despite 
Table 2 Fold change in expression of highly upregulated genes induced by IBV infection

\begin{tabular}{|c|c|c|}
\hline Gene & Description & $\begin{array}{l}\text { Fold } \\
\text { change } \\
\text { (Infected / } \\
\text { Control) }\end{array}$ \\
\hline$I l 2 r g$ & Interleukin 2 receptor subunit gamma & 1.6447 \\
\hline$T f$ & Transferrin (ovotransferrin) & 1.6469 \\
\hline Tlr4 & Toll-like receptor 4 & 1.6504 \\
\hline Il6r & Interleukin 6 receptor & 1.7668 \\
\hline $\operatorname{Tlr} 15$ & Toll-like receptor 15 & 1.7790 \\
\hline$T l r 7$ & Toll-like receptor 7 & 1.8052 \\
\hline Lcpl & Lymphocyte cytosolic protein 1 & 1.8258 \\
\hline Lbp & Lipopolysaccharide binding protein & 1.8319 \\
\hline Ill rap & Interleukin 1 receptor accessory protein & 1.8490 \\
\hline Stabl & Stabilin 1 , transcript variant $\mathrm{X} 2$ & 1.8967 \\
\hline Ilsl2 & Interleukin 8 -like 2 & 1.9669 \\
\hline $\operatorname{Tl} 2 b$ & Toll-like receptor 2 family member B & 1.9793 \\
\hline$C y b b$ & Cytochrome b- 245 beta chain & 1.9834 \\
\hline Mmrll4 & Macrophage mannose receptor 1-like 4 & 2.2063 \\
\hline$C d 48$ & CD48 molecule & 2.2107 \\
\hline Ccl26 & $\mathrm{C}-\mathrm{C}$ motif chemokine ligand 26 & 2.2521 \\
\hline Cclls & Chemokine & 2.3019 \\
\hline $1 l 1 r 2$ & Interleukin 1 receptor type 2 & 2.3726 \\
\hline Ccll7 & Chemokine & 2.3810 \\
\hline$C d 80$ & CD80 molecule & 2.4126 \\
\hline Cd72ag & CD72 antigen & 3.0760 \\
\hline Ccr2 & $\mathrm{C}-\mathrm{C}$ motif chemokine receptor 2 & 3.1495 \\
\hline Marco & $\begin{array}{l}\text { Macrophage receptor with collagenous struc- } \\
\text { ture }\end{array}$ & 3.3576 \\
\hline Selp & Selectin $\mathrm{P}$, transcript variant $\mathrm{X} 2$ & 3.9415 \\
\hline Ccl4 & Chemokine (C-C motif) ligand 4 & 4.0789 \\
\hline$I l 1 b$ & Interleukin 1 , beta & 4.2180 \\
\hline Ccr 7 & $\mathrm{C}-\mathrm{C}$ motif chemokine receptor 7 & 4.2953 \\
\hline Il8l1 & Interleukin 8-like 1 & 4.5621 \\
\hline$A v d$ & Avidin & 5.3144 \\
\hline Il4il & Interleukin 4 induced 1 & 9.7158 \\
\hline
\end{tabular}

the genetic variability of the $\mathrm{S} 1$ gene, the hosts of different IBV strains might use common pathways for protection against the virus. However, it is debatable whether certain genetic modifications contribute significantly to pathogenicity and the host immune response. Although the modification of a few amino acids is enough to affect infectivity [31,32], additional studies are necessary to understand the details of immune regulation. Moreover, our examination suggests the lack of a clear relationship between variations in the S1 protein and the host response. In a phylogenetic analysis of $\mathrm{S} 1$ protein sequences, the genetic distances between K04712 and the M41-CK strain and between K047-12 and the Beau-R strain were similar (Fig. 1A) However, the immune response to K047-12 was more similar to the response to
M41-CK than to the response to Beau-R (Fig. 3A). This observation emphasizes the advantage of transcriptome analysis in host cells over the viral sequence comparisons. Identification of major cytokine pathways that are regulated during acute IBV infection opens paths for determining how adaptive immunity manipulates systemic inflammation. We pinpointed several genes involved in innate immunity. In previous studies, infection with strain M41-CK activated IL-6 and IL- $1 \beta$ production in lung tissue, while an IBV strain with kidney tropism upregulated immune modulators such as IL-6, IL-10RA, IL-17RA, CCL4, and CCL17 [8, 33, 34]. Among the top 20 highly regulated genes, IL-1 $\beta$, IL-10, IL12a, IL-1RAP, IL-2RG, IL-6R, TLR4, TLR7, CCR2, and CCR7 were involved in all of the studies. We confirmed the expression of those genes using real-time PCR and identified IL-1 $\beta$ and IL-10 as signature genes. Produced by mesangial cells, IL-10 is a critical cytokine for pathophysiology in renal disorders. Despite its suppressive effect on inflammation, IL-10 can be upregulated during nephritis when inflammatory lymphocytes must be controlled and induction of regulatory T cells is required $[35,36]$. Along with IL-6, $\mathrm{IL}-1 \beta$ is secreted from renal mesangial cells and mediates inflammation. These are key activators for Th17 cell generation that develops in numerous renal disorders [37, 38].

During acute infection, TLR activation turns on intracellular signals to stimulate proinflammatory cytokines and their receptors, followed by generation of effector $\mathrm{T}$ cells, which is critical for protection, but excessive cytokine secretion is detrimental to the host [39-41]. For example, TLR3 and TLR7 are critical mediators of RNA virus recognition and initiation of the innate immune response [33]. TLR3 and TLR4 are also activated in SARS coronavirus infection $[42,43]$. Considering that TLR4 and TLR7 are upregulated by K047-12 infection, we speculate that IBV might share common features with betacoronaviruses in the regulation of PRR recognition. Existing knowledge from rodent and human immunology about the regulation of chemokine receptors was applied to chickens. CCR2 receives the CCL2 signal and activates monocyte migration for viral clearance, and its deficiency is therefore fatal during infection [44]. CCR7 is activated by CCL19 and CCL21, and its critical role in $\mathrm{T}$ cell migration has been demonstrated in chickens [45]. In addition, human patients with SARS coronavirus infections showed activation of CCR 2 and CCR7 expression $[46,47]$. We also observed CCR2 and CCR7 activation by K047-12, suggesting that the chicken immune system activates chemokine receptors analogous to those in mice and humans and directs immune cell migration and host protection.

Genetic modifications in the IBV genome can result in altered tissue tropism, which makes it difficult to save animals from endemic outbreaks. IBV strains with renal tropism are lethal because they attack both urogenital and systemic 
organs [48]. Therefore, to understand the immune responses in the target organs to viral infection, we performed a transcriptome analysis of primary renal cells and found that the genetic profile in immunologic functions for pathogen recognition, cytokine regulation, and signaling is closely associated with innate immune responses during viral infection. This study provides information about immune pathways that are activated by infection with avian viruses. Expanding our basic knowledge about avian immunology is important for vaccine development and characterization of new variant viruses.

Author contributions Conceptualization: KHM, PJ. Data curation: LR, JJS, PJ. Formal analysis: LR, JJS, YJ, PJ. Investigation: LR, JJS, PJ. Methodology: LR, JJS, JY, PJ. Project administration: KHM, PJ. Supervision: PJ. Validation: LR, JJS, PJ. Writing — original draft: LR, JJS, PJ.

Funding This research was supported by funding from the Office of Research Support, Kangwon National University, a 2020 Research Grant from Kangwon National University (no. 520200065), and a National Research Foundation of Korean (NRF) grant funded by the Korea government (MSIT) (no. 2020R1G1A1099715).

\section{Declarations}

Conflict of interest The authors declare that they have no conflicts of interest.

\section{References}

1. Dinan AM et al (2019) Comparative analysis of gene expression in virulent and attenuated strains of infectious bronchitis virus at subcodon resolution. J Virol 93(18):e00714-19

2. Zhu J et al (2020) Infectious bronchitis virus inhibits activation of the TLR7 pathway, but not the TLR3 pathway. Arch Virol 165(9):2037-2043

3. Li F (2016) Structure, function, and evolution of coronavirus spike proteins. Annu Rev Virol 3(1):237-261

4. Belouzard $\mathrm{S}$ et al (2012) Mechanisms of coronavirus cell entry mediated by the viral spike protein. Viruses 4(6):1011-1033

5. Cavanagh D, Davis PJ, Adrian Mockett AP (1988) Amino acids within hypervariable region 1 of avian coronavirus IBV (Massachusetts serotype) spike glycoprotein are associated with neutralization epitopes. Virus Res 11:141

6. Martinez N et al (2012) Molecular and phylogenetic analysis of bovine coronavirus based on the spike glycoprotein gene. Infect Genet Evol 12(8):1870-1878

7. Cavanagh D (2003) Severe acute respiratory syndrome vaccine development: experiences of vaccination against avian infectious bronchitis coronavirus. Avian Pathol 32(6):567-582

8. Cong F et al (2013) Transcriptome analysis of chicken kidney tissues following coronavirus avian infectious bronchitis virus infection. BMC Genom 14:743

9. Chong KT, Apostolov K (1982) The pathogenesis of nephritis in chickens induced by infectious bronchitis virus. J Comp Pathol 92(2):199-211

10. Cavanagh D (2007) Coronavirus avian infectious bronchitis virus. Vet Res 38(2):281-297
11. Hyun-Woo Moon HWS, Hyuk MK (2019) Genomic characteristics of natural recombinant infectious bronchitis viruses isolated in Korea. Korean J Vet 59:123

12. Kumar S et al (2018) MEGA X: molecular evolutionary genetics analysis across computing platforms. Mol Biol Evol 35(6): 1547-1549

13. Hennion RM, Hill G (2015) The preparation of chicken kidney cell cultures for virus propagation. Methods Mol Biol (Clifton, N.J.) 1282:57-62

14. Bolger AM, Lohse M, Usadel B (2014) Trimmomatic: a flexible trimmer for Illumina sequence data. Bioinformatics 30(15):2114-2120

15. Kim D, Langmead B, Salzberg SL (2015) HISAT: a fast spliced aligner with low memory requirements. Nat Methods 12(4):357-360

16. Pertea $\mathrm{M}$ et al (2015) StringTie enables improved reconstruction of a transcriptome from RNA-seq reads. Nat Biotechnol 33(3):290-295

17. Li H et al (2009) The Sequence Alignment/Map format and SAMtools. Bioinformatics 25(16):2078-2079

18. Raudvere U et al (2019) g:Profiler: a web server for functional enrichment analysis and conversions of gene lists (2019 update). Nucleic Acids Res 47(W1):W191-W198

19. Roh HJ et al (2013) Evaluation of infectious bronchitis virus Arkansas-type vaccine failure in commercial broilers. Avian Dis 57(2):248-259

20. de Wit JJS, Cook JK, van der Heijden HM (2011) Infectious bronchitis virus variants: a review of the history, current situation and control measures. Avian Pathol 40(3):223-235

21. Jackwood MW et al (2007) Molecular and serologic characterization, pathogenicity, and protection studies with infectious bronchitis virus field isolates from California. Avian Dis 51(2):527-533

22. Kuo L et al (2000) Retargeting of coronavirus by substitution of the spike glycoprotein ectodomain: crossing the host cell species barrier. J Virol 74(3):1393-1406

23. Park JY et al (2005) Variations in the nucleocapsid protein gene of infectious bronchitis viruses isolated in Korea. Virus Genes 31(2):153-162

24. Song JE et al (2013) Sequencing, phylogenetic analysis, and potential recombination events of infectious bronchitis viruses isolated in Korea. Virus Genes 46(2):371-374

25. Armesto M, Cavanagh D, Britton P (2009) The replicase gene of avian coronavirus infectious bronchitis virus is a determinant of pathogenicity. PLoS ONE 4(10):e7384

26. Yang $X$ et al (2018) Induction of innate immune response following introduction of infectious bronchitis virus (IBV) in the trachea and renal tissues of chickens. Microb Pathog 116:54-61

27. Yan S-H et al (2016) Pathogenicity of a TW-like strain of infectious bronchitis virus and evaluation of the protection induced against it by a QX-like strain. Front Microbiol 7:1653-1653

28. Kameka AM et al (2014) Induction of innate immune response following infectious bronchitis corona virus infection in the respiratory tract of chickens. Virology 450:114-121

29. Ghetas AM et al (2016) Kidney cell-adapted infectious bronchitis virus Arkansas Delmarva poultry industry vaccine confers effective protection against challenge. Avian Dis 60(2):418-423

30. Abdollahi $\mathrm{H}$ et al (2020) Coronavirus: proteomics analysis of chicken kidney tissue infected with variant 2 (IS-1494)-like avian infectious bronchitis virus. Arch Virol 166:1-13

31. Cavanagh D, Elus M, Cook J (1997) Relationship between sequence variation in the S1 spike protein of infectious bronchitis virus and the extent of cross-protection in vivo. Avian Pathol 26(1):63-74

32. Lin S-Y, Chen H-W (2017) Infectious bronchitis virus variants: molecular analysis and pathogenicity investigation. Int J Mol Sci 18(10):2030 
33. Wang $X$ et al (2006) Transcriptome of local innate and adaptive immunity during early phase of infectious bronchitis viral infection. Viral Immunol 19(4):768-774

34. Okino $\mathrm{CH}$ et al (2014) Inflammatory and cell-mediated immune responses in the respiratory tract of chickens to infection with avian infectious bronchitis virus. Viral Immunol 27(8):383-391

35. Sinuani I et al (2013) Role of IL-10 in the progression of kidney disease. World J Transpl 3(4):91

36. Park $\mathbf{J}$ et al (2016) Chronically elevated levels of short-chain fatty acids induce $\mathrm{T}$ cell-mediated ureteritis and hydronephrosis. J Immunol 196(5):2388-2400

37. Afsar B et al (2018) The future of IL-1 targeting in kidney disease. Drugs 78(11):1073-1083

38. Tashiro $M$ et al (2016) IL-1 $\beta$ promotes tubulointerstitial injury in MPO-ANCA-associated glomerulonephritis. Clin Nephrol 86(4): 190

39. Seo SH et al (2000) Adoptive transfer of infectious bronchitis virus primed alphabeta $\mathrm{T}$ cells bearing CD8 antigen protects chicks from acute infection. Virology 269(1):183-189

40. Mangalmurti N, Hunter CA (2020) Cytokine storms: understanding COVID-19. Immunity 53:19

41. Akira S, Sato S (2003) Toll-like receptors and their signaling mechanisms. Scand J Infect Dis 35(9):555-562

42. Okabayashi $\mathrm{T}$ et al (2006) Cytokine regulation in SARS coronavirus infection compared to other respiratory virus infections. J Med Virol 78(4):417-424

43. Totura AL et al (2015) Toll-like receptor 3 signaling via TRIF contributes to a protective innate immune response to severe acute respiratory syndrome coronavirus infection. MBio 6(3):e00638-15

44. Lim JK et al (2011) Chemokine receptor Ccr2 Is critical for monocyte accumulation and survival in west nile virus encephalitis. $\mathrm{J}$ Immunol 186(1):471-478
45. Annamalai T, Selvaraj RK (2011) Chemokine receptor CCR7 and CXCR5 mRNA in chickens following inflammation or vaccination. Poult Sci 90(8):1695-1700

46. Wong CK et al (2004) Plasma inflammatory cytokines and chemokines in severe acute respiratory syndrome. Clin Exp Immunol 136(1):95-103

47. Cheung CY et al (2005) Cytokine responses in severe acute respiratory syndrome coronavirus-infected macrophages in vitro: possible relevance to pathogenesis. J Virol 79(12):7819-7826

48. Jackwood MW, de Wit S (2020) Infectious bronchitis. Diseases of poultry. Wiley, Oxford, pp 167-188

49. Rohde $\mathrm{F}$ et al (2018) Characterization of chicken tumor necrosis factor- $\alpha$, a long missed cytokine in birds. Front Immunol 9:605

50. Truong AD et al (2017) Chicken IL-26 regulates immune responses through the JAK/STAT and NF- $\mathrm{KB}$ signaling pathways. Dev Comp Immunol 73:10-20

51. Zhou Z et al (2013) Upregulation of chicken TLR4, TLR15 and MyD88 in heterophils and monocyte-derived macrophages stimulated with Eimeria tenella in vitro. Exp Parasitol 133(4):427-433

52. Annamalai T, Selvaraj R (2010) Chicken chemokine receptors in T cells isolated from lymphoid organs and in splenocytes cultured with concanavalin A. Poult Sci 89(11):2419-2425

Publisher's Note Springer Nature remains neutral with regard to jurisdictional claims in published maps and institutional affiliations. 